\title{
On the modelling of valve point loadings for power electricity dispatch
}

\author{
Eric S. Fraga ${ }^{\mathrm{a}, *}$, Lingjian Yang ${ }^{\mathrm{a}}$, Lazaros G. Papageorgiou, ${ }^{\mathrm{a}, *}$ \\ ${ }^{a}$ Centre for Process Systems Engineering (CPSE) \\ Department of Chemical Engineering \\ University College London (UCL)
}

\begin{abstract}
Modelling the effect of valve point loadings on the performance and cost of power generators for electricity dispatch problems is necessary. For the past 20 years, the development of computer based methods for the identification of optimal designs have been based on a single model, introduced by Walters \& Sheble (1993). This model approximates the non-monotonic incremental cost curve using a sine function. This note explores the properties of this model, highlighting one critical deficiency for use within an automated optimisation based design system and proposes a new model.
\end{abstract}

Keywords: power dispatch, valve loadings, optimization, mixed integer linear programming

\section{Modelling valve point loading effects}

Modelling valve point loadings is necessary to capture the losses incurred due to the throttling of partially open valves in electric power generators. Walters \& Sheble 11 introduced an extension to the cost model previously used for dispatch problems to cater for this effect. The model extended the quadratic formulation through the addition of a sine function term:

$$
F_{i}\left(P_{i}\right)=a_{i} P_{i}^{2}+b_{i} P_{i}+c_{i}+\left|e_{i} \sin \left(f_{i}\left(P_{i, \min }-P_{i}\right)\right)\right|
$$

where $F_{i}\left(P_{i}\right)$ is the cost of producing power $P_{i}$ in unit $i$. Each unit has a different cost function, as defined by the parameters $a_{i}, b_{i}, c_{i}, e_{i}$ and $f_{i}$. This model has been used extensively subsequently; see [2] for an overview and [3, 4, 5] for more recent results.

The graph of the cost function for the first unit in a 10 unit case study [6] is shown in Figure 1. The dashed curve shows the cost function as it would be without valve point loading effects taken into account, i.e. $e_{i}$ and $f_{i}$ identically 0 . Although this curve appears linear in this graph, this is due to the small

\footnotetext{
*Corresponding author
} 


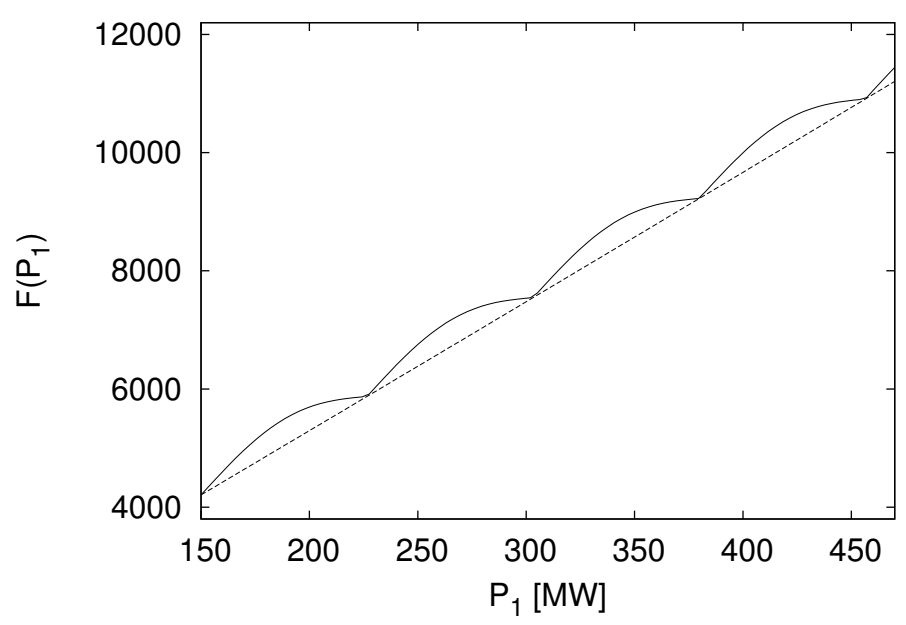

Figure 1: Valve point loading function (solid line) with the equivalent function without valve point loading for comparison (dashed line). The coefficients for the cost function are $a=$ $0.00043, b=21.60, c=958.20, e=450, f=0.041$, and $P_{\min }=150$. Due to small value of the coefficient for the quadratic term, the dashed line appears almost linear.

value of the $a_{i}$ coefficient. For every case study in the literature, most of the cost curves behave in a similar fashion.

The solid curve in Figure 1 represents the full cost model including the effect of valve point loadings. This graph exhibits the qualitative behaviour we expect. The opening of a valve leads to a greater fuel load with the effect tailing off as the valve opens further. This is described in the literature as a non-monotonic incremental change.

However, for some units, we find that not only is the incremental change nonmonotonic, the actual fuel load is also non-monotonic: there are cases where the fuel load curve has a negative slope. This leads to a negative valued incremental cost curve, suggesting that the cost for an increase in power output decreases. For instance, Figure 2 shows the graph of equation 1 for unit 7 from the same case study [6] with the graph of the slope of this equation shown along the bottom. Negative values of the slope are clearly visible and cover a significant percentage of the domain of operation for this particular unit.

\section{A gedanken experiment}

Cost curves with negative incremental values appear in all the case studies considered for valve point loading effects in the literature. Analysis of the results presented indicates that the solutions, i.e. the loadings on the units, are often very close to the valve points. Given the negative incremental cost curves and these solutions, we wonder whether the solutions obtained are an artifact of the 


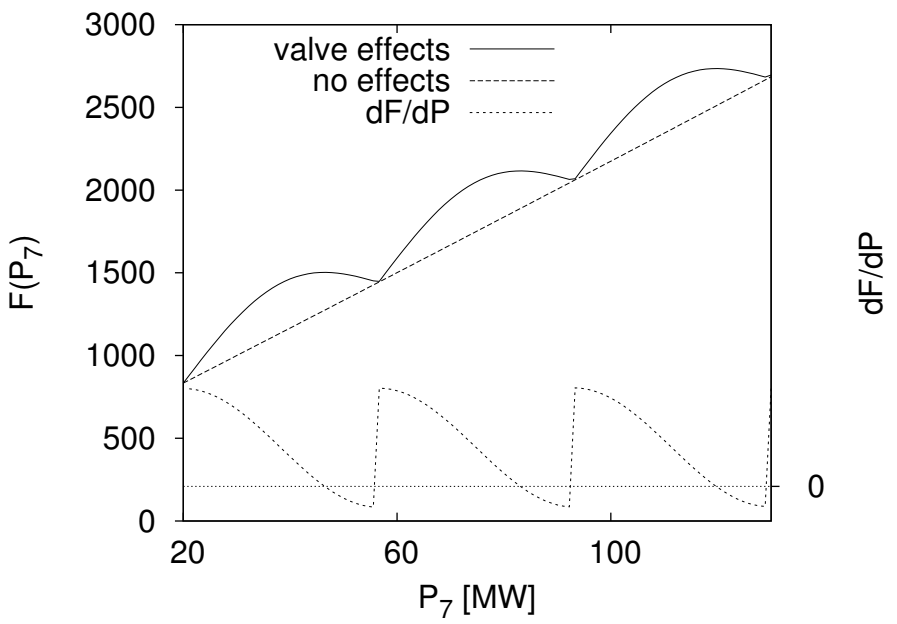

Figure 2: Valve point loading function (solid line) with the equivalent function without valve point loading for comparison (dashed line) for unit 7 in the 10 unit example [6]. The coefficients for the cost function are $a=0.00211, b=16.51, c=502.70, e=300$ and $f=0.086$. The curve with shorter dashes at the bottom of the graph, corresponding to the right $y$ axis, shows the slope of the function with valve opening effects with the negative values highlighted by the horizontal dotted line indicating the 0 value for the slope.

models. We propose a simple deterministic model and solution procedure to investigate this conjecture.

\subsection{A mixed-integer linear programming model}

The model we propose is the original cost model without valve point loading effects but with the search space restricted to power loads only at the valve points for all of the units, i.e. the points at which valves are fully open. The optimisation problem, in this case, becomes:

$$
\min _{Y_{i, j}} z=\sum_{i=1}^{n}\left[a_{i} \sum_{j} L_{i, j}^{2} Y_{i, j}+b_{i} \sum_{j} L_{i, j} Y_{i, j}+c_{i}\right]
$$

where the right hand side is simply the sum of the costs of each unit. The decision variables are represented by $Y_{i, j}$, a set of binary variables, with $i=$ $1, \ldots, n$ identifying the $n$ different units and $j=1, \ldots, j_{i, \max }$ the choice of the different valve point loading points for each unit $i$. For each unit, only one operating point can be chosen:

$$
\sum_{j} Y_{i, j}=1 \quad \forall i=1, \ldots, n
$$


The optimisation goal is to identify the operation of each unit, restricted to the valve point loadings, to meet the requirements, specifically a given overall demand $P_{D}$ :

$$
\begin{array}{rlrl}
P_{i} & =\sum_{j} L_{i, j} Y_{i, j} & \forall i=1, \ldots, n \\
\sum_{i=1}^{n} P_{i} & \geq P_{D}
\end{array}
$$

The load on each unit, $P_{i}$, is chosen from the set of discrete loads possible, $L_{i, j}$, defined by the valve points. These load points are calculated a priori and enumerated using the index $j$ :

$$
\begin{aligned}
L_{i, j} & =P_{i, \min }+(j-1) \frac{\pi}{f_{i}} \quad j=1, \ldots, j_{i, \max } \\
j_{i, \max } & =\underset{j}{\arg \max }\left\{L_{i, j} \leq P_{i, \max }\right\}
\end{aligned}
$$

$j_{i, \text { max }}$ is the number of valve points for each unit $i$, defined by the domain of operation for each unit and the value of $f_{i}$.

As we are constraining the power outputs to those possible with the units operating only at the valve loading points, the power demand may not be met exactly. Instead, we constrain the problem to deliver at least as much power as required but possibly more. This will lead to solutions that may be more expensive than is required but these will be solutions that are feasible.

The resulting model is a mixed integer linear programme (MILP) which we will refer to as DVL_MILP (discrete valve loading MILP) below. The careful use of binary variables, $Y_{i, j}$, enables us to write the original quadratic cost function as a linear combination of the binary variables with pre-determined load points, noting that, at the valve points, the extra term introduced by Walters \& Sheble 1 is identically 0 and so need not be included. The advantage of an MILP model is the ability to find, efficiently and with guaranteed convergence, the best solution possible within the restricted search spaced defined by the valve points alone.

\subsection{Results}

DVL_MILP has been implemented in GAMS [7] and solved using the CPLEX solver. In all cases, solutions are obtained in a fraction of a second on a typical desktop computer.

The model has been applied to three case studies from the literature. The first two are both a 13 unit problem, with two different demands [8]. Three of the 13 units, the first, second and fourth units, in this problem have negative slope cost curves due to the valve point effect modelling. The third case study is a 40 unit problem [9] in which 19 of the units exhibit cost function curves with negative slopes due to the valve point effect modelling. 
Table 1 shows how the solutions obtained by the restricted search model compare with the best solutions known for these problems [4, 5]. The results are compared in terms of the actual power output and the cost. For presentation purposes, the costs have been rounded to the nearest unit. We see that even though the search space has been restricted significantly, the solutions obtained compare favourably. The solutions obtained by DVL_MILP are, at most, $0.5 \%$ away from the best known solution in each case, for both the cost and the power delivered measures.

Table 1: Gap between solutions obtained with the restricted search MILP model and the best known solutions from the literature.

\begin{tabular}{lrrrrrr} 
Problem & Demand & $\begin{array}{r}\text { DVL_MILP } \\
\text { output } \\
(\mathrm{MW})\end{array}$ & $\begin{array}{r}\text { Excess } \\
\text { output } \\
(\%)\end{array}$ & $\begin{array}{r}\text { DVL_MILP } \\
\text { cost }\end{array}$ & $\begin{array}{r}\text { Best } \\
\text { known } \\
\text { cost }\end{array}$ & $\begin{array}{r}\text { Gap } \\
(\mathrm{MW})\end{array}$ \\
\hline 13 units & 1800 & 1802 & 0.11 & 17964 & 17960 & 0.02 \\
13 units & 2520 & 2525 & 0.20 & 24174 & 24164 & 0.04 \\
40 units & 10500 & 10501 & 0.01 & 121986 & 121413 & 0.47
\end{tabular}

These results provide support for the notion that the valve point effect models used in the literature may be leading to misleading results. However, from an engineering point of view, it may make sense to operate all units with valves fully open, or as close to that as possible. On the other hand, this may exacerbate the impact of valve opening in a dynamic context. These issues lead us to argue for improved models for valve point loadings, especially for the dynamic context as addressed by Chiou [6].

\section{Concluding remarks}

This short note has presented some observations on the cost functions used in the literature to model the impact of valve-point loadings. Many of the examples in the literature exhibit non-monotonic incremental cost curves. As a thought experiment, we have also presented a new approximate MILP model that can provide good quality solutions by only allowing for designs at the valve points. This model is robust and fast so is well suited for real-time management applications or as a basis for an initialisation step for other approaches, such as those based on meta-heuristics.

Robert K Green \& Shahriar Sahba, in the discussion of the paper by Noyola et al. [10, noted:

"[...] the availability of accurate incremental heat rate data is essential for the proper execution of economic dispatch calculation programs."

This highlights a difficulty we have found in addressing problems in this area: the data used to generate the incremental cost curves to take into account valve 
point loading effects are not in the open literature. It is difficult to compare the functions proposed to the original data. Further, it is not possible to tune the models and their parameters to avoid the problem of negative incremental cost curves. We suggest that this be an area that should be explored more fully.

\section{References}

[1] D. C. Walters, G. B. Sheble, Genetic algorithm solution of economic dispatch with valve point loading, IEEE Transactions on Power Systems 8 (3) (1993) 1325-1332.

[2] N. Sinha, R. Chakrabarti, P. K. Chattopadhyay, Evolutionary programming techniques for economic load dispatch, IEEE Transactions on Evolutionary Computation 7 (1) (2003) 83-94.

[3] T. Niknam, A new fuzzy adaptive hybrid particle swarm optimization algorithm for non-linear, non-smooth and non-convex economic dispatch problem, Applied Energy 87 (1) (2010) 327-339.

[4] L. dos Santos Coelho, V. Cocco Mariani, An efficient cultural selforganizing migrating strategy for economic dispatch optimization with valve-point effect, Energy Conversion and Management 51 (12) (2010) $2580-2587$.

[5] T. Niknam, H. D. Mojarrad, H. Z. Meymand, A novel hybrid particle swarm optimization for economic dispatch with valve-point loading effects, Energy Conversion and Management 52 (4) (2011) 1800-1809.

[6] J.-P. Chiou, A variable scaling hybrid differential evolution for solving largescale power dispatch problems, IET Generation, Transmission \& Distribution 3 (2) (2009) 154-163.

[7] A. Brooke, D. Kendrick, A. Meeraus, R. Raman, GAMS: A user's guide, GAMS Development Corporation, Washington (1998).

[8] L. dos Santos Coelho, V. C. Mariani, Combining of chaotic differential evolution and quadratic programming for economic dispatch optimization with valve-point effect, IEEE Transactions on Power Systems 21 (2) (2006) 989-996.

[9] A. I. Selvakumar, K. Thanushkodi, Optimization using civilized swarm: Solution to economic dispatch with multiple minima, Electric Power Systems Research 79 (2009) 8-16.

[10] A. H. Noyola, W. M. Grady, G. L. Viviani, An optimized procedure for determining incremental heat rate characteristics, IEEE Transactions on Power Systems 5 (2) (1990) 376-383. 